\title{
A Novel Intelligent Unified Controller for the Management of the Unified Power Flow Controller (UPFC) using a Single Back Propagation Feed Forward Artificial Neural Network
}

\author{
K. A. Rani Fathima ${ }^{1 *}$ and T. A. Raghavendiran ${ }^{2}$ \\ 'Department of Electrical \& Electronics Engineering, Sathyabama University, Chennai, \\ Tamil Nadu, India; fathima_powersystems@yahoo.co.in \\ ${ }^{2}$ Anand Institute of Higher Technology, Chennai, Tamil Nadu, India
}

\begin{abstract}
Since the advent of the UPFC there have been immense developments, based on control systems associated with the management of the UPFC. Traditionally the PI, Fuzzy and Neural Network control techniques have been in used for the four control units of the UPFC. This paper presents the design and development steps of a novel control scheme for the UPFC. The proposed technique believes that a single combined ANN based controller can be trained appropriately to handle all the mappings associated with the inputs and the corresponding outputs associated with all the controllers of the UPFC. It is demonstrated in this paper by way of simulations in the MATLAB / SIMULINK environment how a single ANN can be developed and used in place of the four controllers usually used in the management of the UPFC. The observations of enhanced performance in terms of improved dynamic behaviour by the usage of a single ANN control strategy are presented in this paper.
\end{abstract}

Keywords: Artificial Neural Network, Fuzzy Logic Controller, Unified Power Flow Controller (UPFC) PI

\section{Introduction}

In a Single Machine Infinite Bus bar (SMIB) system the Unified Power Flow Controller (UPFC) can be incorporated and well utilised for the purpose of enhanced power flow from the single machine to the grid. The grid, in this case is the load for the single machine. The core rationale behind the facts devices is to increase the power transaction capabilities as high as possible just limited by the thermal limits of the corridor and the terminal equipments. The UPFC offers a set of useful features that can provide a comprehensive complete control over the power transaction requirements.
With the help of the UPFC, the real, reactive power flow, magnitude and angle of receiving end voltage and line impedance can be modified according to the requirements by power economic conditions prevailing and changing from time to time. Followed by the initial and innovative contributions by N.G. Hingorani ${ }^{1}$ on Flexible AC Transmission Systems (FACTS), the UPFC was first formulated by Gyuagi in the year $1992^{2,3}$.

Since then there have happened a number of developments on three important aspects of the basic UPFC equipment. The major developments that have taken place in the UPFC system are a. Topological changes like increased voltage levels of converters. b. Variations

${ }^{*}$ Author for correspondence 
in PWM generation for the converters like SPWM, SVPWM, SHE PWM etc. and c. Control system variations like PI, PID, Fuzzy Logic Control, Artificial Neural Network (ANN) based control, Hysteresis control etc. Apart from these variations for the design of the UPFC, variations in mathematical models have also been widely reported in the literature. Of all these the most important is the appropriate generation of the reference signal that modulates the discretely switched power electronic converters. The reference signal embeds the actual dictation as to how the power converters should behave.

Despite the fact that there have been volumes of literature presented and available related to the design and control of the UPFC, in reality the number of worldwide industrial implementations of the UPFC are not appreciable and the fact that the complexity in the design and the control strategies of the UPFC may be one among the other reasons for the noticeable limited usage of the UPFC by the electricity distribution and transmission departments and other industries. The entire global scenario exhibits only a few, just countable, UPFC installations.

$\mathrm{In}^{4}$ the authors have presented control scheme using fuzzy logic controllers. Four fuzzy logic controllers have been used to manage the UPFC. In real time applications when realised in micro controllers or microprocessors, since there are four fuzzy logic controllers, it leads to large timing over heads.

$\mathrm{In}^{5}$ the authors have presented a generalised investigation of the different control schemes to manage the UPFC. The problem with the PI based control scheme is that it requires hectic mathematical procedures. Tuning of the four PI controllers becomes very tedious at the same time the performance becomes a handicap when approximate models are used.

$\operatorname{In}^{6}$ the authors have presented a control scheme using PI controllers and the tuning is done with Genetic algorithm. Genetic algorithm based tuning leads to optimised control still it is not robust as only few parameters have been considered in the development of the fitness function.

Particle swarm optimisation has been tried for the management of UPFC wide ref $f^{7}$. Though it works well in the cases where the solution is in the neighbourhood but if the global solution is far from the search end the PSO struck up with the local solution which may not be all that acceptable.

Several other ${ }^{8-9}$ general purpose, heuristic optimisation algorithms have also been reported. But these models require a dedicated computer and the solutions are arrived at consuming more time and as such these schemes are not suitable for real time applications.

A consideration of the different design schemes as reported in the literature can reveal that the single ANN based control scheme is the next natural evolutionary choice.

In this research it is attempted that a simple comprehensive all ANN and a single ANN based control scheme is developed and presented. The design methodology of the single ANN based control scheme is elaborated with a comparison of the results of Fuzzy Logic and PI control schemes. The MATLAB / SIMULINK based simulation of the proposed scheme reveals that the single ANN based control scheme outperforms the PI, fuzzy logic and the individual ANN model of control in the four controllers used in the UPFC, in terms of dynamic performance.

While four ANN control units, for the four controllers in total, with two controllers each for the two converters of the UPFC are reported in the literature, in this paper a single combined ANN scheme is proposed. This innovation is inspired by the fact that while individual ANN units can be trained on different sections of control, then it is quite possible that a single ANN unit can do it as well, and this idea could be more practical, easily verifiable and feasible in real time applications since trained ANNs can be easily implemented in real time using high speed, high resolution modern micro controllers with large memory as well.

The combined ANN model is trained using the training data set derived from the individual ANN units. Therefore the single ANN can comprehend the whole system as it is the trained combined model of the individual ANNs. Combined single ANN has the advantage that the total numbers of neurons are reduced since the hidden layer becomes common for all the inputs and outputs mapping. That is a number of neural networks with few inputs and few outputs now becomes a single ANN unit with more inputs and outputs but with a common hidden layer.

Four different versions of control schemes have been studied and compared. A PI based control scheme, an FLC based scheme, four ANN control model and finally the single ANN model are compared in terms of power transaction, power factor, voltage profile and THD of the voltage at the point of common coupling.

Next to the introduction given in chapter 1 the rest of the paper is arranged in the following manner. Chapter 
II gives a simple treatment of the control preliminaries of the UPFC. Chapter III presents the design of the PI and fuzzy logic based control schemes. Chapter IV gives an overview of the ANN based control scheme as proposed in this work. The results are compared in chapter $\mathrm{V}$ followed by the conclusion.

\section{The UPFC}

The objectives, structure and the simplified mathematical relations of the UPFC are discussed in this chapter.

a. Any reactive power requirement to be supplied by the single machine to the grid whether continuous or sudden will be supplied by the UPFC thus relieving the single machine from loading with reactive power demands.

b. Sudden and short duration requirements of real power at the grid will be met out from the DC link Capacitor, which the single machine may not be able to cope up in time.

c. The voltage across the grid will be maintained by the series converter of the UPFC by injecting a series voltage in the appropriate phase and magnitude at conditions of voltage variations across the grid.

d. The voltage across the point of common coupling will be maintained by injecting appropriate quantity of reactive power by the shunt converter. This relieves the single machine from reactive power burden and this can maintain single machine side power factor as well at a higher value close to unity. e. And finally for the proper functioning of all these features mentioned above an appropriate DC link voltage is to be maintained at the DC link.

\subsection{General Structure of the UPFC}

The UPFC is the integration of two power converters integrated through a common DC link. The DC link carries an energy storage device typically a capacitor. The AC side of the shunt converter is connected across the AC mains at the point of common coupling through a reactor and also a voltage matching transformer. The AC side of the series converter is connected to a three phase transformer the secondary of which comes in series with the AC line feeding the grid. The arrangement of an UPFC is as shown in Figure 1.

\subsection{The Shunt Converter}

The shunt converter is a three leg three phases Graetz bridge converter with a DC side and a three phase AC side. The three phase AC side is connected across the three phase AC transmission lines at the PCC through a voltage transformer and a series reactor as shown in Figure 2.The purpose of the shunt converter is to inject reactive power into the bus bar. It works according to Kirchoff's current law, it injects current in parallel into the Point of Common Coupling (PCC). The simplified vector diagram of currents is shown in Figure 3. Reactive power injection is carried out by injecting three phase reactive currents synchronised with the line. A PWM generator generating synchronised switching pulses switch the six switches of the three legs shunt converter.

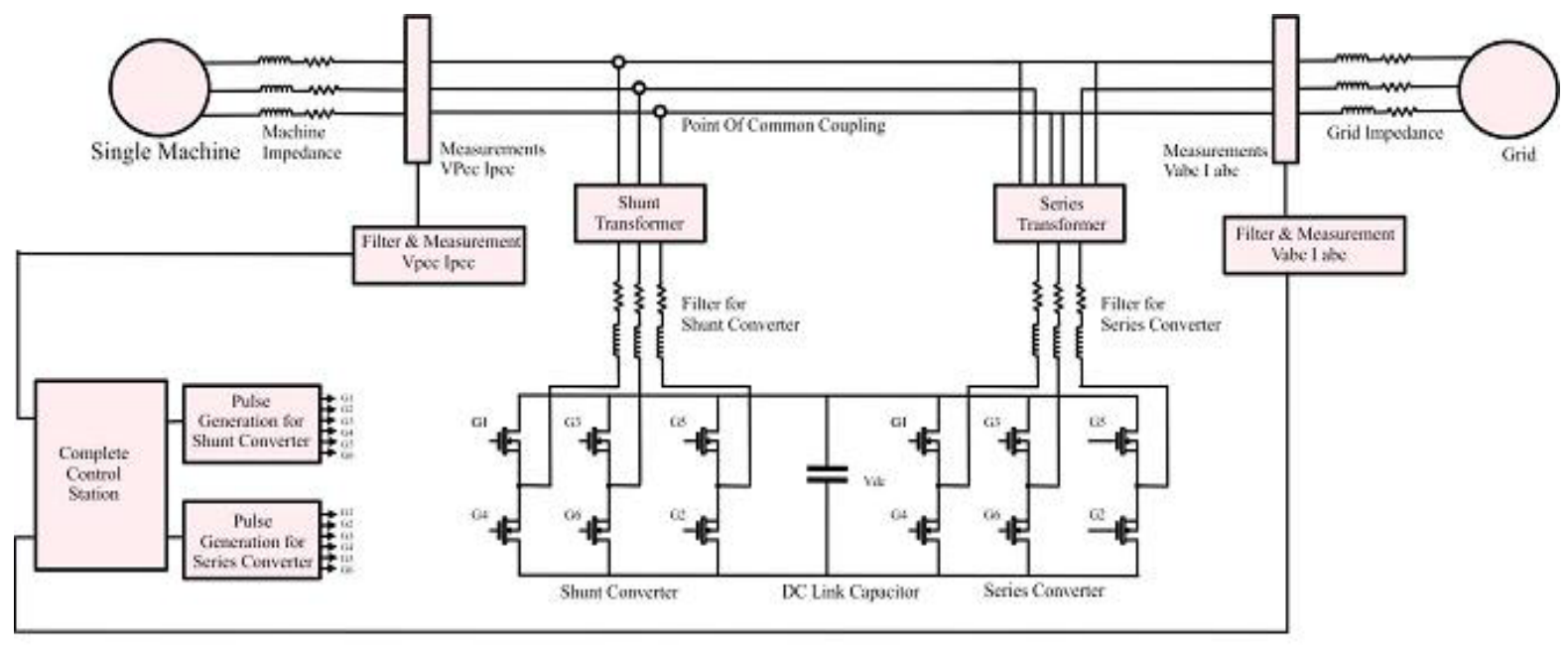

Figure 1. Block Schematic of UPFC. 


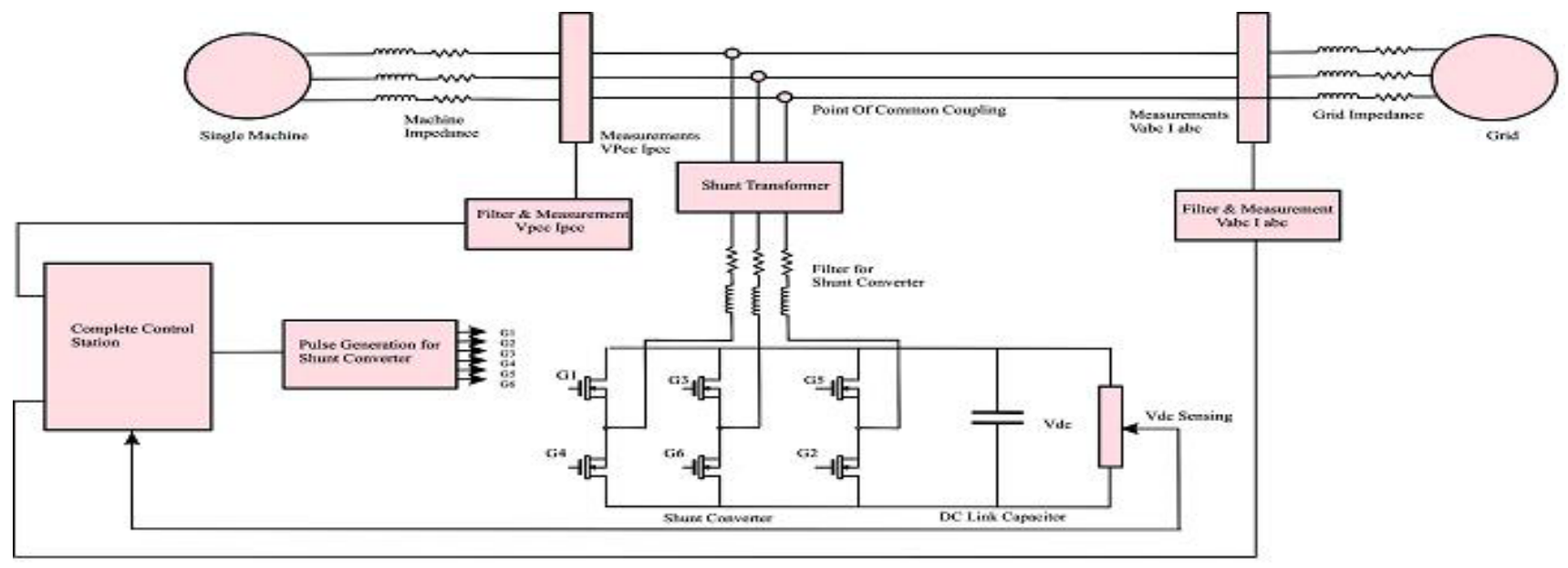

Figure 2. The Statcom Unit.

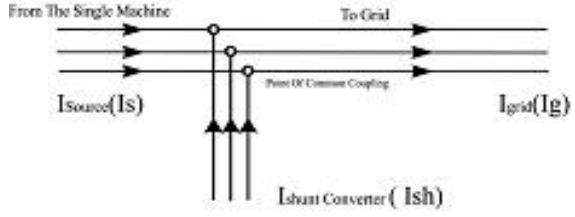

Simplified Vector Diagram ( Ish)

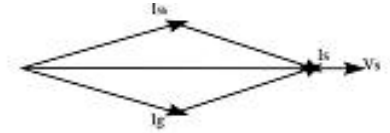

Simplified Vector Diagram ( Ish)

Figure 3. Vector Diagram of the Currents of the Statcom.

The objectives of the shunt convertor can be achieved by appropriately supplying the switching pulses. A three phase reference signal is first created according to the control law to be adopted. The generation of the switching pulses is governed by the three phase reference signal that is produced by the contribution of two controllers. The control objective of injecting appropriate reactive power into the bus bar is thus accomplished by the proper generation of the reference signal with the help of two controllers.

\subsection{Reactive Power Transaction}

Consider two nodes A and B, linked by a reactor, between which we need reactive power transaction as shown in Figure 4. As for reactive power transaction between the two nodes $\mathrm{A}$ and $\mathrm{B}$ connected by the reactor $\mathrm{X}$ it is required that the voltage at node $\mathrm{A}$ be higher in amplitude than that at node $\mathrm{B}$ such that reactive power can flow from node $A$ to $B$ and vice versa.

This happens according to the equation (1).

$$
Q=\left(\frac{V_{1}\left(V_{1}-V_{2}\right)}{X}\right) \operatorname{Cos} \delta
$$
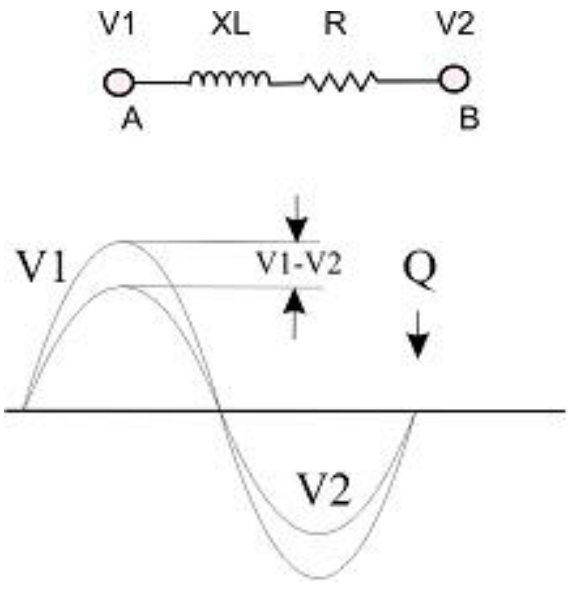

Figure 4. Reactive power flow by difference of voltage.

Where V1 and V2 are the two nodal voltages and X being the reactance between them then if the two nodal voltages are in phase, then $\operatorname{Cos} \delta$ becomes equal to $\operatorname{Cos} 0$ $=1$ and for the given potential difference between $\mathrm{V} 1$ and V2 the reactive power transaction is maximum. Higher reactive power transaction happens with higher difference in the nodal voltages as shown in Figure 4. In the shunt converter, therefore, the control of the modulation 
index can control the AC side voltage of the converter which appears at one end of the reactor. The other end of the reactor is at Vpcc stepped down by the transformation ratio of the voltage matching transformer.

\subsection{Real Power Transaction}

The DC side of the shunt converter carries a fixed capacitor and this capacitor is to be held at a constant DC potential. This DC potential across the DC link capacitor is maintained by the shunt converter. For this purpose the shunt converter is associated with real power transaction in the process of maintaining the required DC voltage across the DC link capacitor.

The real power transaction between the PCC and the DC link capacitor happens through the Shunt Converter and its operation is governed by the following equation.

$$
P=\frac{V_{1} V_{2} \operatorname{Sin} \delta}{X}
$$

Real power transaction between two nodes at voltages V1 and V2 and linked by a reactor $\mathrm{X}$ is maximized when the phase angle V1 and V2 is 90 degrees as shown in Figure 5. With 90 degrees phase difference between V1 and V2, the factor Sin $\delta$ in equation 2 becomes Sin $90=1$.

Under certain circumstances the energy stored in the DC link capacitor is to be utilised by the series converter for supplying real power to the load. A sudden real power demand met by the series converter utilises the stored energy of the capacitor and whenever this happens the voltage across the DC link capacitor falls down and this is made up by the shunt converter by drawing the required real power from the AC bus

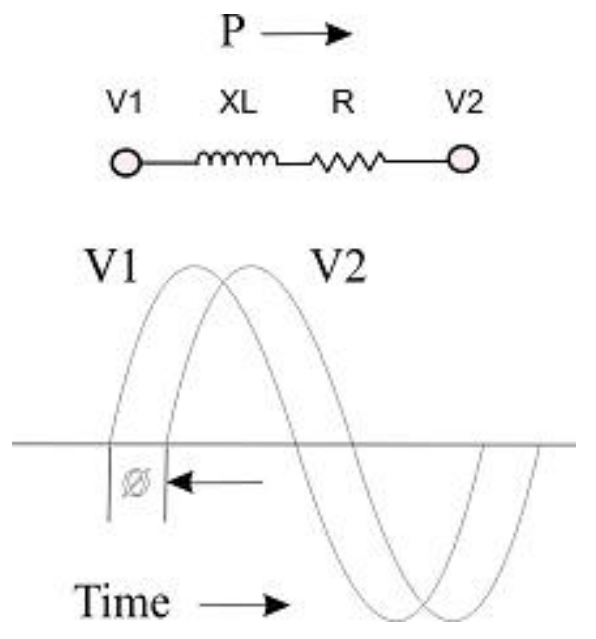

Figure 5. Real power flow with difference in phase angle. and restore the DC link voltage across the DC link capacitor.

The duties of the shunt converter can be consolidated as

a. To inject the required reactive power into the PCC.

b. To maintain DC link voltage at a constant level.

\subsection{The Controllers Associated with the Shunt Converter}

The two duties of the shunt converter as depicted earlier are carried out successfully with the help of two controllers associated with the shunt converter. A block diagram showing the position of the two controllers along with their input and output signals are shown in Figure 6.

\subsection{The Series Converter}

The purpose of the series converter is to insert a supplementary voltage along with the main $\mathrm{AC}$ voltage thus inject real power into the bus bar. It works according to Kirchoff's voltage law; it inserts voltage in series, at appropriate magnitude and angle along with the bus voltage as shown in Figure 7.
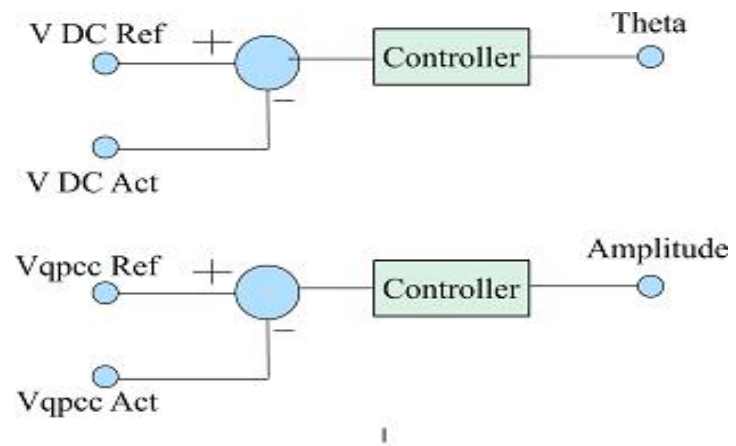

Figure 6. A general control structure for the Statcom.

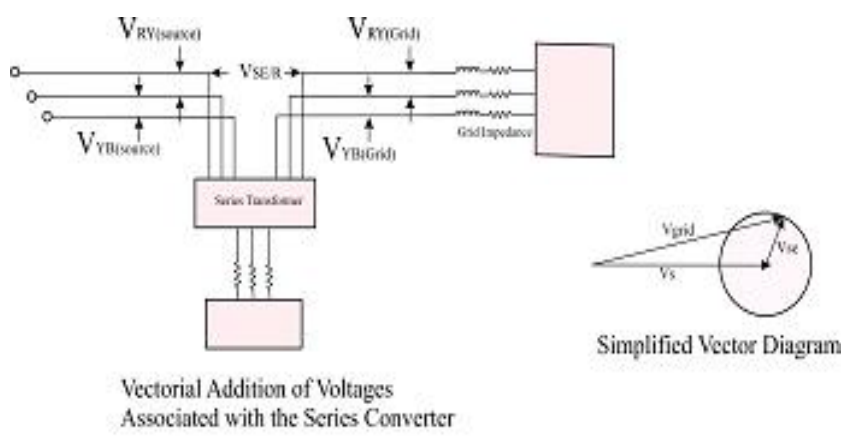

Figure 7. Block diagram of the series converter and vector diagram of the series converter. 
The series converter is a three leg three phases Graetz bridge converter with a DC side and a three phase AC side. The three phase AC side is connected across a three phase transformer and the secondary of the transformer comes in series with the AC bus bar as shown in Figure 8.

Supplementary Real power insertion is carried out by injecting three phase voltage of appropriate magnitude and phase along with the transmission bus bar voltage. A PWM generator generating synchronised but appropriately phase shifted switching pulses switch the six switches of the three leg shunt converter. The objective of the series convertor can be achieved by appropriately supplying the switching pulses. A three phase reference signal is first created according to the control law to be adopted. The generation of the switching pulses is governed by the three phase reference signal that is produced by the contribution of two controllers. The control objective of inserting appropriate real power into the bus bar is carried out by the proper generation of the reference signal with the help of two controllers.

\subsection{Real Power Transaction in Series Converter}

The DC side of the series converter carries a fixed capacitor, shared by the shunt converter as well, and this capacitor is to be held at the appropriately required constant DC potential. This DC potential across the DC link capacitor is maintained by the shunt converter. For this purpose the shunt converter is associated with real power transaction in the process of maintaining the required DC voltage across the DC link capacitor.

During periods of sudden real power the series converter complements the main power source. The series converter output voltage with certain magnitude and angle with respect to the bus bar AC voltage adds up

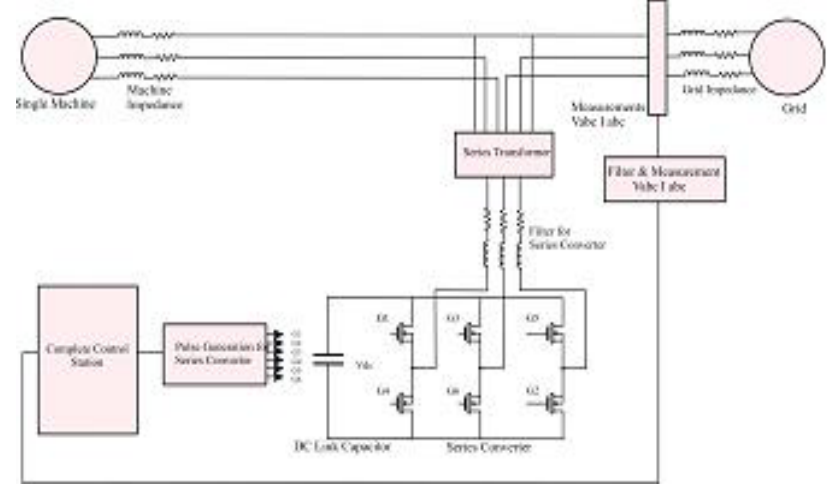

Figure 8. Position of the Series Converter. vectorially with the bus bar AC voltage. Thus the resultant of these two voltages will be the receiving end voltage as in Figure 7.

A fall in the voltage across the load side can be made up by adjusting the series injected voltage derived from the series converter. The series converter while makes up the required AC bus voltage, by series insertion of voltage delivers the corresponding real power and reactive power as applicable to that part of the injected voltage.

\subsection{The Controllers Associated with the Series Converter}

The series converter ensures two requirements. The real and reactive power demand of the load must be met with. For this purpose the ' $\mathrm{d}$ ' and ' $\mathrm{q}$ ' component voltages at the terminal under consideration should be maintained. Maintaining $\mathrm{Vd}$ and $\mathrm{Vq}$ ensures the real and reactive power demands. The two duties of the series converter of maintaining $\mathrm{Vd}$ and $\mathrm{Vq}$ as per the set points are carried out successfully with the help of two controllers associated with the series converter. A block diagram showing the position of the two controllers along with their input and output signals are shown in Figure 9.

\section{The PI and Fuzzy Logic Control Implementations for the UPFC}

The variables and the mathematical relations associated with the Single Machine Infinite Bus Bar system are given below:

$\mathrm{V}_{\mathrm{s}}$ is the Single Machine side Terminal voltage

$I_{s}$ the Current drawn from the Single machine.

$\mathrm{V}_{\text {se }}$ is the Voltage inserted in series.
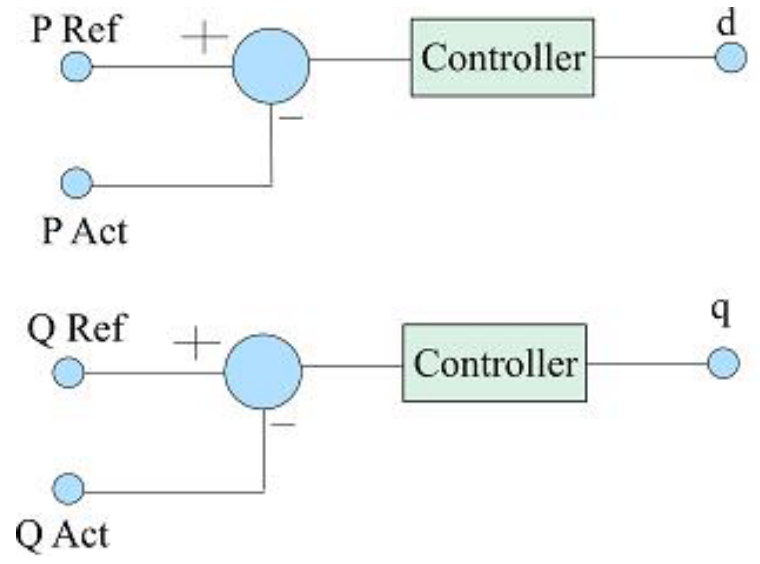

Figure 9. General control structure for the series converter. 
$\mathrm{I}_{\mathrm{sh}}$ is the shunt converter injected current.

$V_{R}$ the Grid side voltage or the receiving end voltage.

$I_{s}+I_{s h}$ is the current drawn by the grid.

The quantities $\mathrm{V}_{\mathrm{s}}, \mathrm{V}_{\mathrm{r}}, \mathrm{V}_{\mathrm{sh}}, \mathrm{V}_{\mathrm{se}}, \mathrm{I}_{\mathrm{s}}$, and $\mathrm{I}_{\mathrm{sh}}$ are all vector quantities.

The series and the shunt converters share a common DC link voltage and the three phase voltage output of these converters are decided and governed by the modulation index MI and phase angle of the reference signal and are related by the expression shown.

The system under consideration, as shown in Figure 1 consists of a single machine and an infinite bus bar, wherein the UPFC is located close to the single machine.

The enhancement in power transferability of the single machine, with the aid of the UPFC is studied. The performance of the system with and without UPFC is studied.

The shunt converter injects the current $I_{s h}$ such that the reactive current requirement of the load is not drawn from the single machine thus improving the source side power factor and the voltage at the PCC.

The series converter can ensure the existence of the required voltage across the load leading to the meeting out of the actual real and reactive power demands at the load.

The shunt converter acts as a controlled current source and its output current should be sufficient to support the reactive power demand of the grid. The reactive power demand of the grid would otherwise burden on the single machine.

\subsection{The DQ Transformation}

The quantities of interest like the source side three phase voltages and the load side voltages are sinusoidal in nature. And these quantities are to be converted into representative DC quantities and then used in the controllers. The simplified model of UPFC is shown in Figure 10.

The conversion of the three phase voltages denoted as $\mathrm{V}_{\mathrm{abc}}$ into $\mathrm{V}_{\mathrm{d}}$ and $\mathrm{V}_{\mathrm{q}}$ in the rotating frame is known as $\mathrm{DQ}$

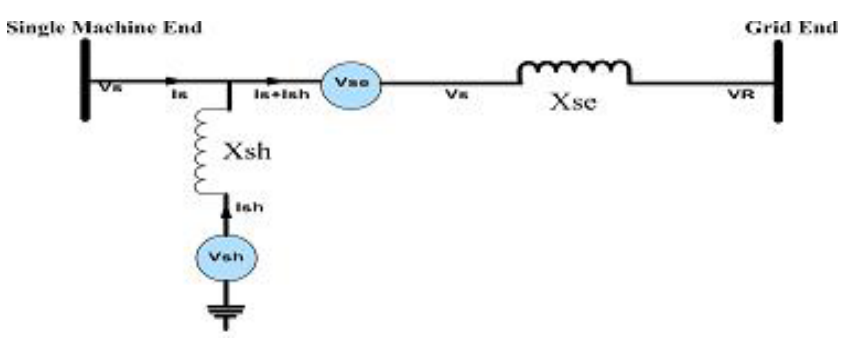

Figure 10. Simplified model of the UPFC. transformation. The vector $\left[\begin{array}{lll}v_{a} & v_{b} & v_{c}\end{array}\right]$ can be transformed into another vector $\left[v_{d} v_{q} v_{0}\right]$ with the help of a transformation matrix.

It can be viewed that

$$
\begin{aligned}
& {\left[\begin{array}{l}
v_{d} \\
v_{q} \\
v_{0}
\end{array}\right]=\frac{2}{3}\left[\begin{array}{ccc}
\sin \theta & \sin \left(\theta-\frac{2 \pi}{3}\right) & \sin \left(\theta+\frac{2 \pi}{3}\right) \\
\cos \theta & \cos \left(\theta-\frac{2 \pi}{3}\right) & \cos \left(\theta+\frac{2 \pi}{3}\right) \\
\frac{1}{2} & \frac{1}{2} & \frac{1}{2}
\end{array}\right]\left[\begin{array}{l}
v_{a} \\
v_{b} \\
v_{c}
\end{array}\right]} \\
& {\left[\begin{array}{l}
v_{a} \\
v_{b} \\
v_{c}
\end{array}\right]=\left[\begin{array}{ccc}
\sin \theta & \cos \theta & 1 \\
\sin \left(\theta-\frac{2 \pi}{3}\right) & \cos \left(\theta-\frac{2 \pi}{3}\right) & 1 \\
\sin \left(\theta+\frac{2 \pi}{3}\right) & \cos \left(\theta+\frac{2 \pi}{3}\right) & 1
\end{array}\right]\left[\begin{array}{l}
v_{d} \\
v_{q} \\
v_{0}
\end{array}\right]}
\end{aligned}
$$

The vector $\left[\begin{array}{lll}v_{a} & v_{b} & v_{c}\end{array}\right]$ and the elements of the transformation matrix are time varying but the output vector $\left[v_{d} v_{q} v_{0}\right]$ is not time varying. However any change in the amplitude of either of va or vb or vc is reflected in the vector elements $\left[v_{d} v_{q} v_{0}\right]$.

All the four controllers used in the UPFC use the source and load voltage in the format $\left[v_{d} v_{q} v_{0}\right]$.

\subsection{Control Strategy Adopted in this Research}

The Figure 11 shows the control strategy adopted in the management of the UPFC.

The control strategy adopted is that a set of four Fuzzy Logic Controllers are first developed, each two, for the generation of the two reference signals pertaining to the two converters. Then the data collected from the input and outputs sides of these FLCs are used to train four ANNs individually and four ANNs replace the four fuzzy logic controllers.

In the next stage all the four ANN units are replaced by a single ANN with 8 inputs and four outputs. The finally derived single ANN unit will take care of the entire control operation. To start with a nominal resistive balanced load is connected to the source and the source and load voltages are measured and converted into the $\left[v_{d} v_{q}\right.$ $\left.v_{0}\right]$ format. Thus we get $\left[v_{d} v_{q} v_{0}\right]$ for both source side and load side voltages.

Under this loading condition the voltage across the load is at an acceptable nominal value, the load being resis- 


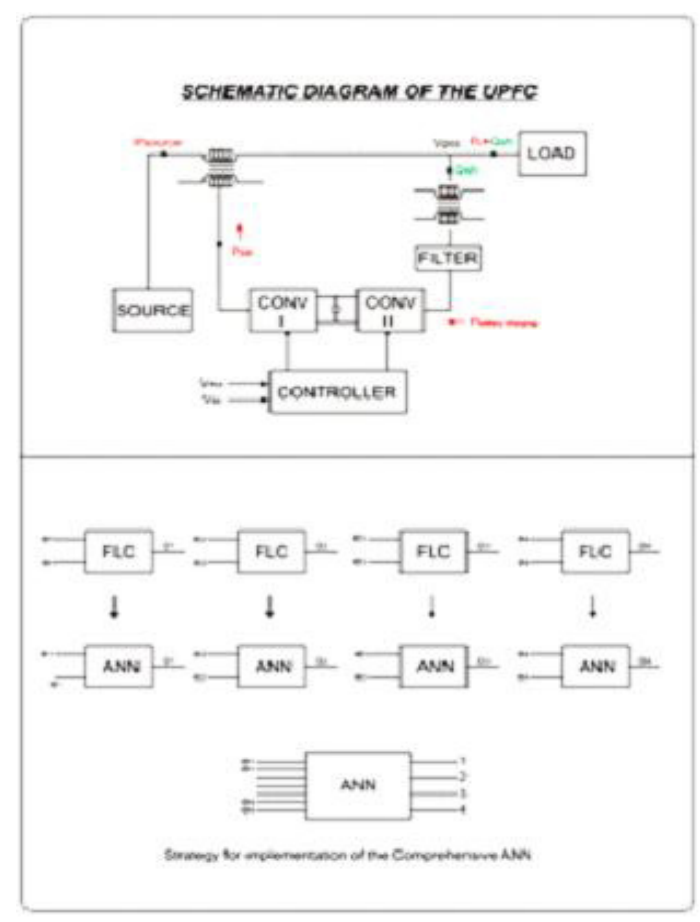

Figure 11. Control strategy adopted in the management of the UPFC.

tive the source side power factor is nearly unity and since the load is linear all voltages and currents are sinusoidal. The objective of the UPFC is to maintain this condition, in the event of faults happening in the single machine side. In other words the objective of the control system is simplified and is just to track this set of $\left[v_{d} v_{q} v_{0}\right]$ at the load and source sides. The additional task of the control system is to maintain the DC link voltage a constant.

Associated with the two converters there are four controllers. The first controller of the shunt converter is used to maintain the DC link voltage; the second controller associated with the shunt converter is used to maintain the AC voltage at the point of common coupling. The other two controllers are associated with the series element of the UPFC and are used to maintain $v_{d}$ and $v_{q}$ at the load terminals. These two controllers can be directly used to monitor the required real and reactive power transmitted to the grid.

\subsection{The Basic Control Schemes of the UPFC}

\subsubsection{The PI Control Scheme}

In this research the UPFC was first designed with a PI control scheme. In the case of the shunt converter the controlled parameters were the DC link voltage and the $\mathrm{d}$ component of the dq transformed three phase voltage at the point of common coupling. In this case the manipulated parameters are respectively the angle theta and the amplitude (Modulation Index) of the reference voltage as shown in Figure 12.

In the case of the series converter the controlled parameters were the real power demand and the reactive power demand. In this case the manipulated parameters are respectively the angle theta and the amplitude of the reference voltage or MI and it is achieved indirectly after reverse transformation from corrected $\mathrm{d}$ and corrected $\mathrm{q}$ back to $V_{a b c}$, which will be the reference wave.

The control scheme block diagram as implemented in MATLAB / SIMULINK environment and the results achieved thereof are illustrated in Figures 13 to 16.

Three performance parameters are considered for analytical comparison with other types of controllers. They are,
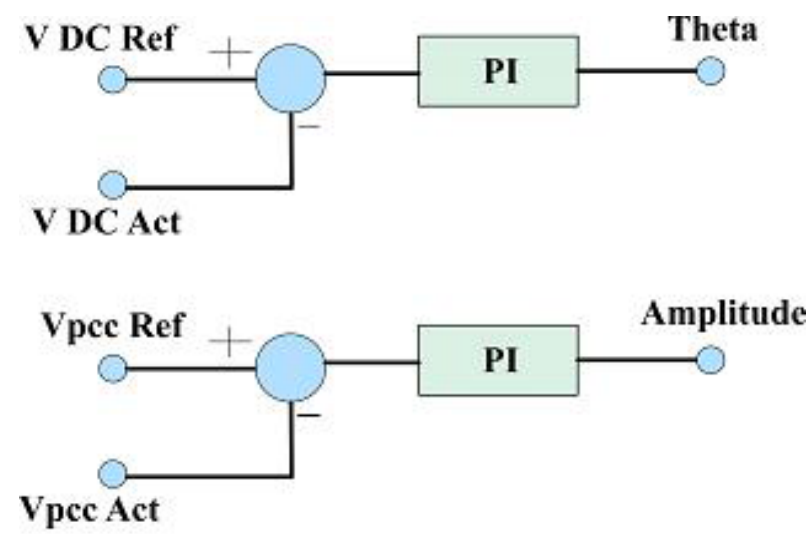

Figure 12. PI controller for the Statcom.

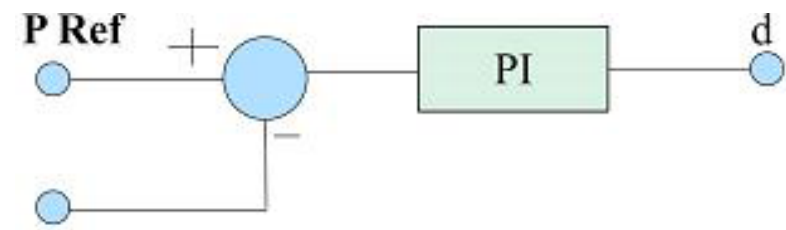

PAct

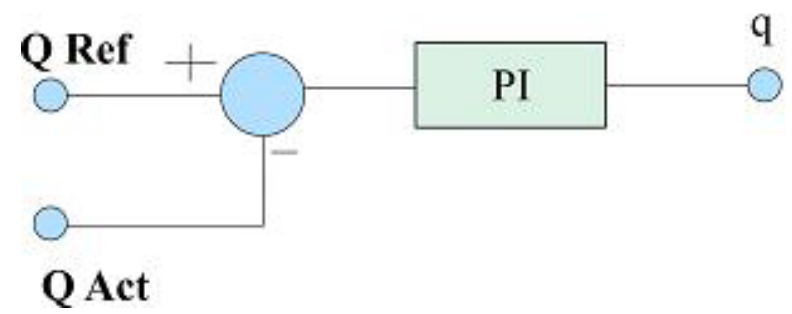

Figure 13. PI controller for the SSSC. 
a. The transient performance of the controlled parameters after the disturbances and commands.

b. The integrated square errors in all the cases.

c. The harmonic performance as represented by THD of the voltage at the PCC and the current drawn from the single machine.

\subsubsection{Fuzzy Logic Control}

The fuzzy logic controller and the ANN based control are two popular intelligent control techniques. Both these schemes of control are inspired by human experience in controlling systems.

As for the fuzzy logic control scheme, it is a technique incorporating human experience, to deal with data in the linguistic form to arrive at an agreeable result. Unlike a PID type of control the Fuzzy logic control scheme does not require a mathematical model and is especially suitable when a precise mathematical model of the system under consideration is either not available or the available mathematical model of the system is very complex. Though at the outset, fuzzy logic control design is not about bringing about a precise control solution, like the precise PID variants, FLCs can be tuned to offer convincing results in areas where the degree of agreeability is wide. The designs of FLCs are quite easy. While mathematical overheads are avoided, they can be intelligent and comprehensive.

The four fuzzy logic controllers adopted in the four sections of controls used in the management of the UPFC are almost identical and are as shown in Figures 14 and 15. They are all of the Mamdani type with two inputs, the error and error rate with one output.

The output of the fuzzy logic controllers contribute for the reference signal generation in terms of the modulation
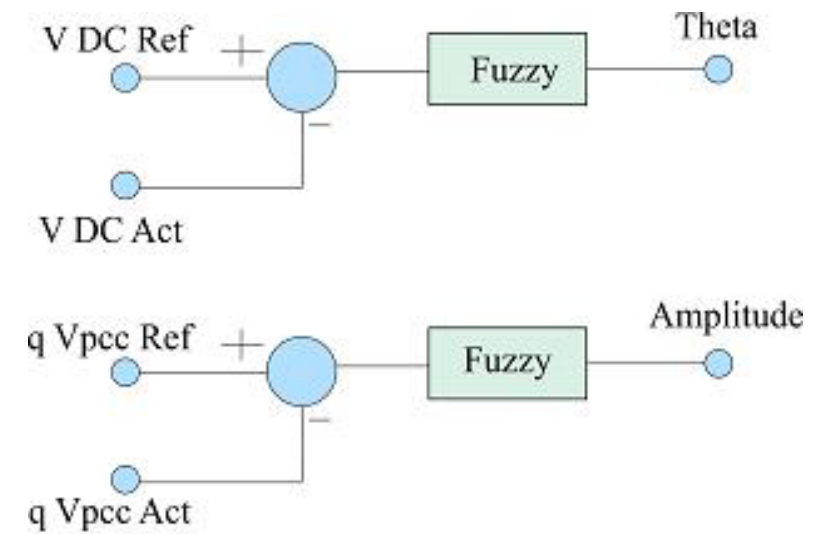

Figure 14. Fuzzy Logic Controller for the Statcom.
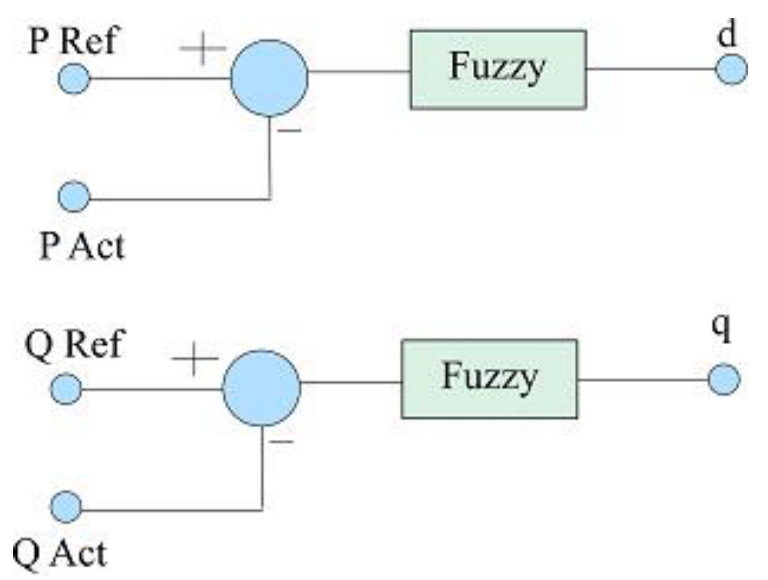

Figure 15. Fuzzy Logic Controller for the Statcom.

index and the phase angle or phase shift. The generated reference will be used for the respective PWM generator units. The details of the FLC controller like the number of segments of the universe of discourse, the shape of the membership functions adopted and the rule matrix are shown in Figures 16-19.

Error and the error rate are the inputs to each of the fuzzy logic controller and each FLC gives the control output corresponding to the input data set. The mapping between the input and the output of the FLC in respect of its rule base are static and time invariant. For the given input set the corresponding output will be dictated according to the rule base adopted.

The point of interest is that there exists a functional mapping between the inputs and the outputs and this mapping is time invariant. As such the FLC can be regarded as a static functional mapping.

The static mapping nature of the FLC can be used to train an ANN and the ANN thus trained can behave exactly like the FLC. (except that, the ANN, with limited number of neurons in the hidden layer can be faster than the FLC when realised in a digital micro controller).

The four FLCs can finally be replaced by four correspondingly trained ANNs.

\section{The Artificial Neural Networks}

The one to one functional relationship between the members of a domain and a co domain can be trained upon the artificial neural Networks. That is the relationship between the input and the output vectors of a system with linear or nonlinear relationships can be trained in an ANN and the ANN can now mimic the functionality 


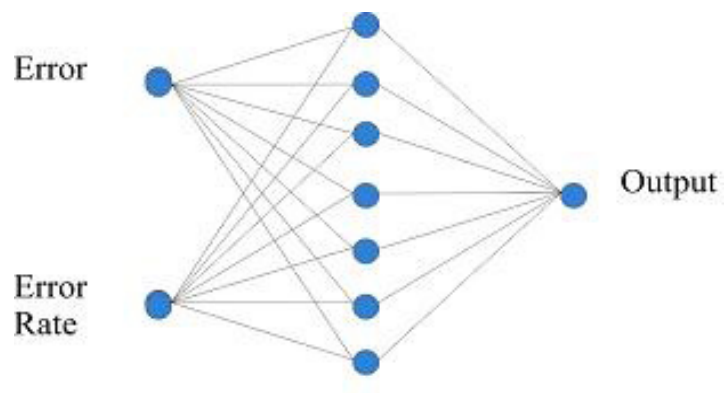

Figure 16. The General Structure of an ANN unit.

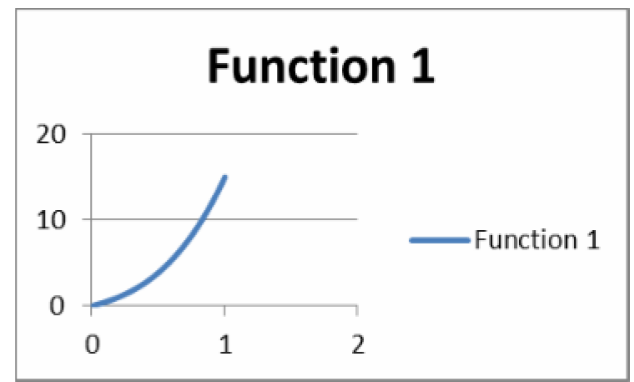

Figure 17. A plot of expression 1 .

of the domain_codomain relationship or simply a system with inputs / outputs.

The general structure of the ANN is shown in Figure 20. ANNs are networks of neurons wherein the neurons are arranged in a number of layers. The layers are named input layer, hidden layer and the output layer. The neurons in the input layer are equal to the number of elements in the input vector. The number of neurons in the output layer is equal to the number of elements in the output vector. In the feed forward back propagation neural network there is at least one hidden layer and optionally more than one hidden layer. In this work the single hidden layer model is used. The number of neurons used in the hidden layer is usually a matter of empirical decisions adjudged by the user. However it is usually more than the number of neurons in the input and output layers individually. In this work each ANN used have two neurons in the input layer, one in the output layer and eleven neurons in the hidden layer, though in Figure 20 less neurons are shown in the hidden layer for the sake of graphical clarity.

The neurons in the input layer are connected individually to all the neurons in the hidden layer. All the neurons in the hidden layer are connected to those in the output layer. When the output of a neuron in one layer is connected as input to any neuron in the next layer, the transmitted signal between the two neurons gets

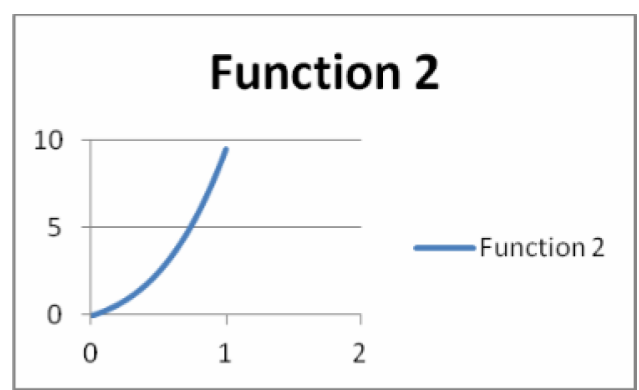

Figure 18. A plot of expression 2.

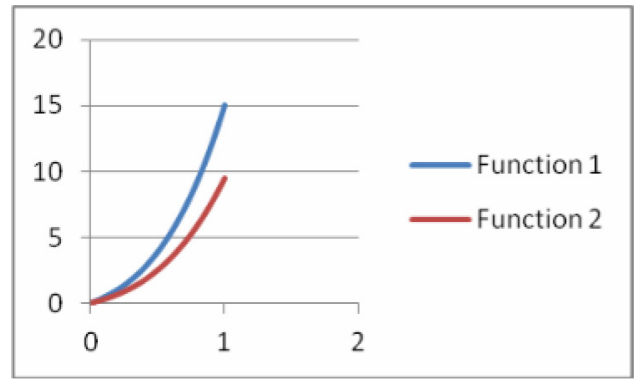

Figure 19. Plot of Expressions 1 and 2.

attenuated or amplified by a factor known as the synaptic weight.

The outputs of all neurons from one layer undergo this linear change, caused by the synaptic weight, before it reaches the receiving neuron.

A neuron can receive some data, process the data and give away an output. Every neuron is thus a processing element. The function of each neuron as a processing element is fixed and can be selected from a set of probable and popular sets of neuronal functions like sigmoidal, signum, purely linear function etc. During the training or testing phases of the ANN the functionality of the neurons does not change. Neurons with different functionality for the different layers are usually acceptable in many applications. However neurons with the same functionality can also be used in all layers as adopted in this case.

Before the ANN gets trained, to start with, the synaptic weights are randomly initialised at the first time formation of an ANN but during the training phase the synaptic weights undergo changes according to the learning rule and finally as the training is over each synaptic weight reaches a final value.

During the test phase or during the period the ANN is put under service to mimic a function, even as the inputs are applied to the ANN the synaptic weights do not change, they just work together with the neurons to give the output. 


\section{Combining ANN Units}

As discussed earlier, an ANN of the feed forward back propagation type, in its basic form has an input layer, a hidden layer and an output layer. There may be more number of hidden layers than one. Let us consider the case of ANNs having only one hidden layer. The style adopted in the combination of the four ANNs is as shown in Figure 20.

Actually as a result of combination of the ANNs the resultant ANN has now more number of inputs and outputs. However the number of neurons in the hidden layer remains the same. Thus all the neural networks combined to form the final single ANN have to share the common hidden layer neurons.

In an attempt to demonstrate how the ANNs are combined in this work the following example may be considered.

Consider two nonlinear equations, wherein $\mathrm{k}$ is an independent variable and let it be time in second.

$$
\begin{array}{cc}
\mathrm{M} 1=(7 *(\mathrm{k} \wedge 3)+4 *(\mathrm{k} \wedge 2)+4 * \mathrm{k}) \\
0.01 & 0.040407 \\
0.02 & 0.081656 \\
0.03 & 0.123789 \\
\ldots \ldots & \ldots \ldots . \\
\ldots \ldots & \ldots \ldots . \\
0.96 & 13.71955 \\
0.97 & 14.03231 \\
0.98 & 14.34994 \\
0.99 & 14.67249 \\
1 & 15 \\
\mathrm{M} 2=(4 *(\mathrm{k} \wedge 3)+3 *(\mathrm{k} \wedge 2)+2.5 * \mathrm{k}) \\
0.01 & 0.025304 \\
0.02 & 0.051232 \\
0.03 & 0.077808 \\
\ldots \ldots & \ldots \ldots . \\
\ldots \ldots & \ldots \ldots . \\
\ldots \ldots . & \ldots \ldots . \\
0.97 & 8.898392 \\
0.98 & 9.095968 \\
0.99 & 9.296496 \\
1 & 9.5
\end{array}
$$

A plot of the two functional relations is as shown in Figure 19.

Now $r$ two independent ANNs can be considered which are trained respectively with the input/output data set, for the range of $\mathrm{k}$ between 0 and 1 in steps of .01 as dictated by each of the two equations. Upon creating and training the two ANNs in the MATLAB / SIMULINK environment two test models were constructed to test the performance of the two ANNs and was found to be as shown in Figures 20a and 20b.

The individual performance of the two ANNs was as recorded in Figures 21a and 21b.

A third ANN was then created with two inputs and two outputs. This is the combined model. For training this ANN model the input data will be the two input vectors which are one and the same that is time $(\mathrm{k})$. The output vectors of model 1 and 2 are now used as the training data as output for the third model

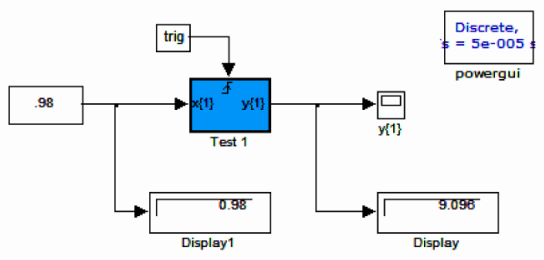

Figure 20a. Test model with the ANN for Expression 1.

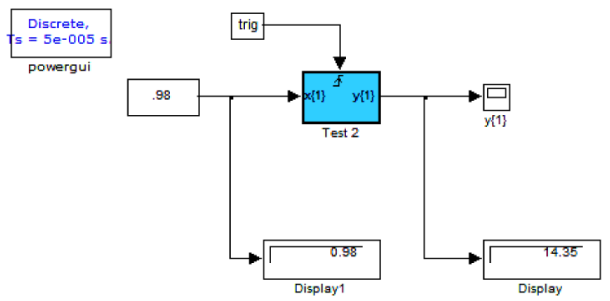

Figure 20b. Test Model with the ANN for Expression 2.

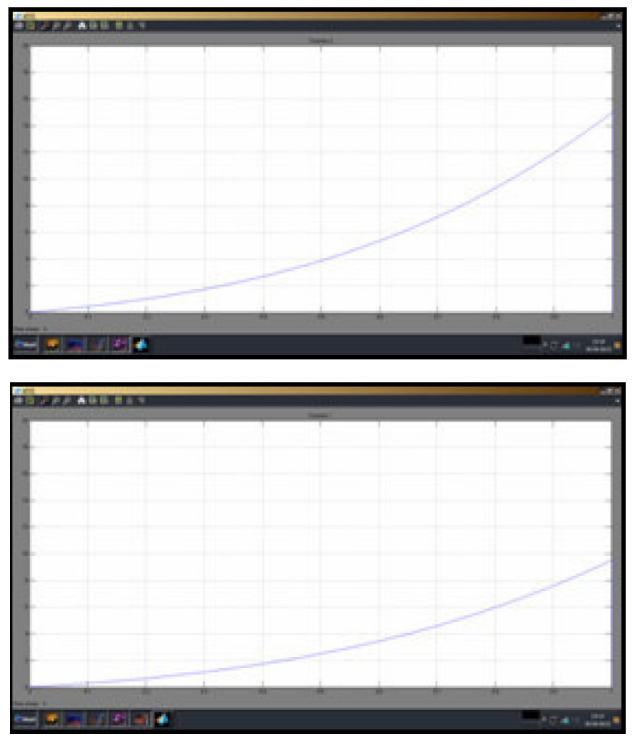

Figure $21 \mathrm{a}$ and $\mathrm{b}$. Individual performance of the two ANNs. 
The third ANN was then trained and a test model as shown in Figure 22 was then formed to test the performance of the third and the final combined ANN model.

The performance of the combined model, as shown in Figure 22, is as good as the performance of the individual ANNs. A plot of the two functional relations for combined model is shown in Figure 23.

To prove that the combined ANN performs better than the individual ones under certain circumstances, if at all, is beyond the scope of this paper. For the context of the paper it is enough that the combined ANN performs as good as the individual ANNs.

What is interesting to note is that the various PI controllers, taking care of different references, but working in a common plant cannot be combined like this. The various FLCs working in a plant for different objectives can neither be combined. But ANNs can be combined without losing the individuality of the one to one mapping of the various inputs and outputs, and this is what this article is all about, as applied to an UPFC.

If there exists a number of ANNs working in a plant each taking care of a particular control section then they can all be combined as a single ANN and this single ANN can take care of the entire plant in maintaining the set points as the individual ANNs were doing. The advantages are twofold. The first being the reduction in the number of hidden layer neurons directly resulting in reduction of code size in processor based implementation or total cell size in VLSI based implementation.

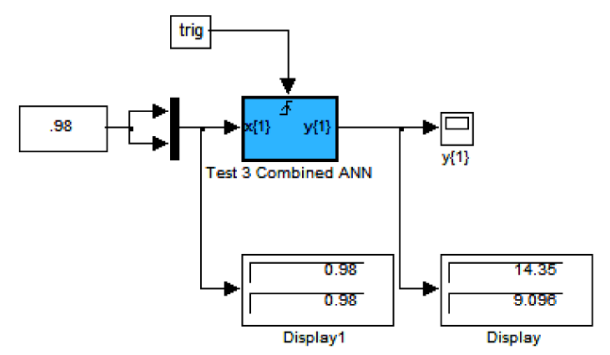

Figure 22. Combined test model for expression 1 and 2.

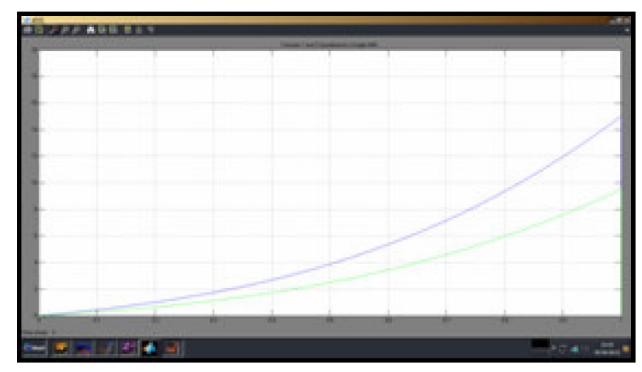

Figure 23. Plot of Expression 1 and 2.
The second advantage is an indirect one. The von Neumann architecture which uses the fetch decode execute cycle, though implemented in a parallel microprocessor system, is a serial method of problem solving. Therefore if many ANN units are involved the time it takes to resolve a problem is much higher when compared to resolving a system with a single neural network.

A trained neural network in terms of its synaptic weights and the activation function can be down loaded into a microprocessor, as used in this work. In doing so the $\mathrm{C}$ program in the Keil programming environment becomes too large if four trained ANNs are to be included. However if a single ANN can replace the coding of the four ANNs the total stuff to be decoded and executed reduces and as a result the speed of operation increases naturally resulting in better dynamic performance of the system under consideration, in the present case the UPFC.

\section{Combining a Number of Neural Network based Controllers}

There are situations like the case of the UPFC where a number of controllers are in action. Some of them may be of any of the variants of the PID, some fuzzy logic and some may be of the ANN type. All controllers can well be replaced by individual ANN based functional mapping schemes for the purpose of control applications. Each of the ANN may have a certain number of inputs and a certain number of outputs depending upon which controller it is replacing.

In this work, four FLCs were first designed, the performance of the overall UPFC system was observed under different loading conditions which could potentially cause disturbances to the power quality. After it was observed that the four FLC control scheme worked satisfactorily, they were replaced by four ANNs and finally a single ANN replaced the four ANNs.

The input and output data required to train the ANNs were the error, error rate and the corresponding output of the FLC as they were collected from the MATLAB workspace when the FLC controlled model was running in the SIMULINK MATLAB environment. The same procedure was simultaneously adopted for collecting the input output data pertaining to other FLCs also.

Using the neural network fitting tool available in MATLAB Neural Network TOOLBOX four ANNs were formed and each trained with the input output data corresponding to each of the four FLCs. 
The UPFC model was now rearranged with the ANNs in place of the FLCs. The performance of the overall UPFC system, being controlled by the four ANNs, was observed under different loading conditions which could potentially cause disturbances to the power quality. After it was observed that the four ANN based control scheme worked satisfactorily, they were replaced by a single ANN.

Finally a single neural network was formed with eight inputs and four outputs. The structural scheme is as shown in Figure 24.

The input and output training data required to train the single ANN were the inputs and outputs associated with each of the four ANNs as they were collected from the MATLAB workspace when the four ANN model was running in the SIMULINK MATLAB environment.

After removing the four ANNs the UPFC model was now rearranged with the single ANN and the inputs and outputs associated with the four ANNs are now routed through the single ANN. The performance of the overall UPFC system, being controlled by the single ANN was observed under different loading conditions which could potentially cause disturbances to the power quality.

The various recordings of the parameters are presented in Figures 31-34.

\section{Results and Discussions}

The different control methodologies like PI, Fuzzy, multiple ANN scheme, and the single ANN scheme were studied in the MATLAB / SIMULINK environment and the performance parameters were compared. Simulink diagram for the proposed schemes are shown in Figures 25-30.

The important parameters compared were the basic power system requirements like real and reactive power delivered to the load. In addition the source side power factor, voltage at the point of common coupling, the integrated square error between the expected voltage at PCC
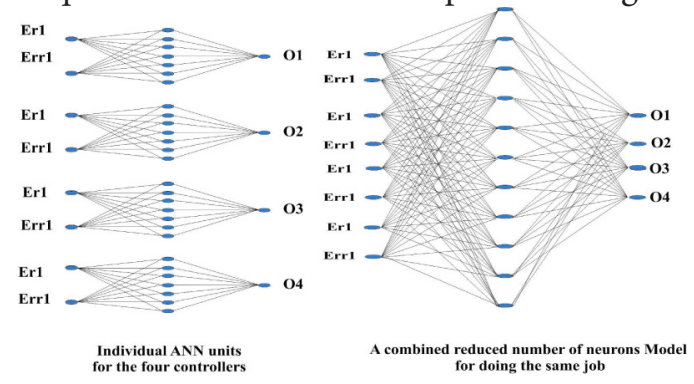

Figure 24. The evolution of the single ANN model from four ANN units.

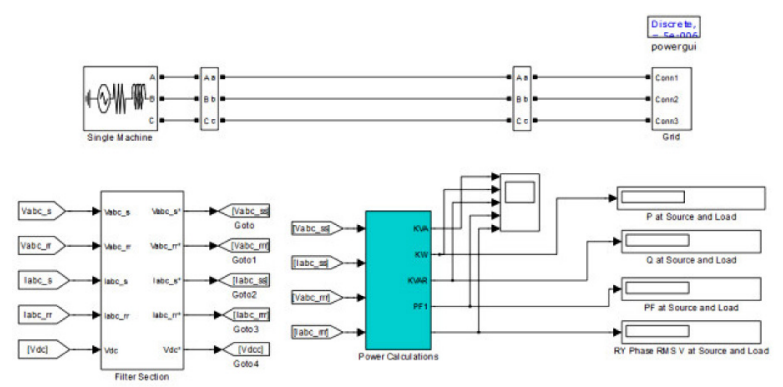

Figure 25. A single machine connected to an Infinite bus bar of a grid.

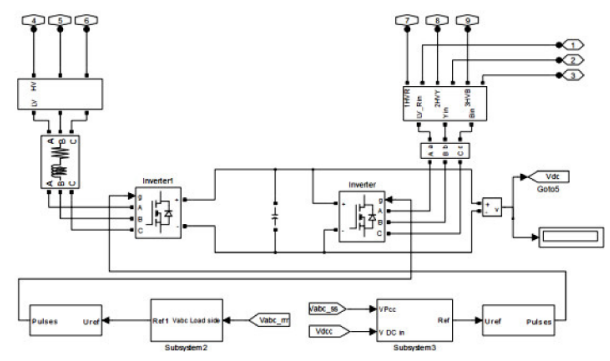

Figure 26. The UPFC with the two converters and their controllers.

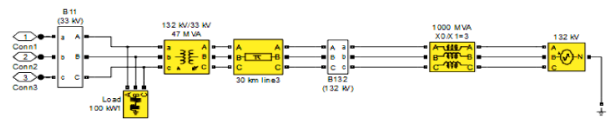

Figure 27. The grid under consideration.

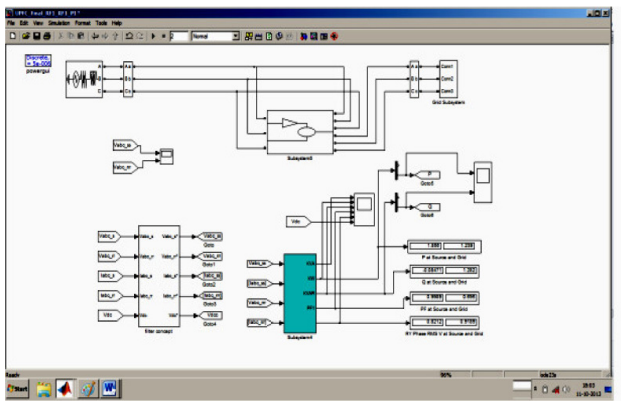

Figure 28. The position of the UPFC in the SMIB system.

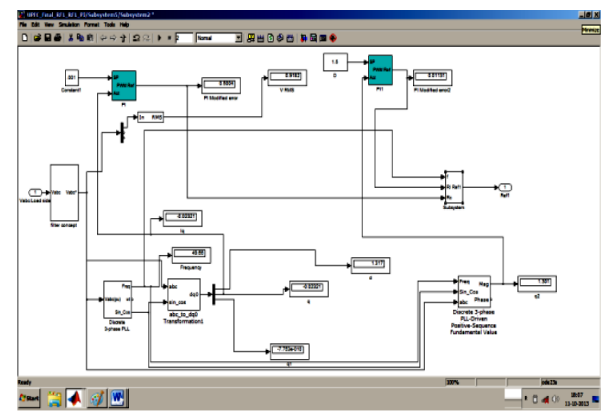

Figure 29. The controllers associated with the series converter. 


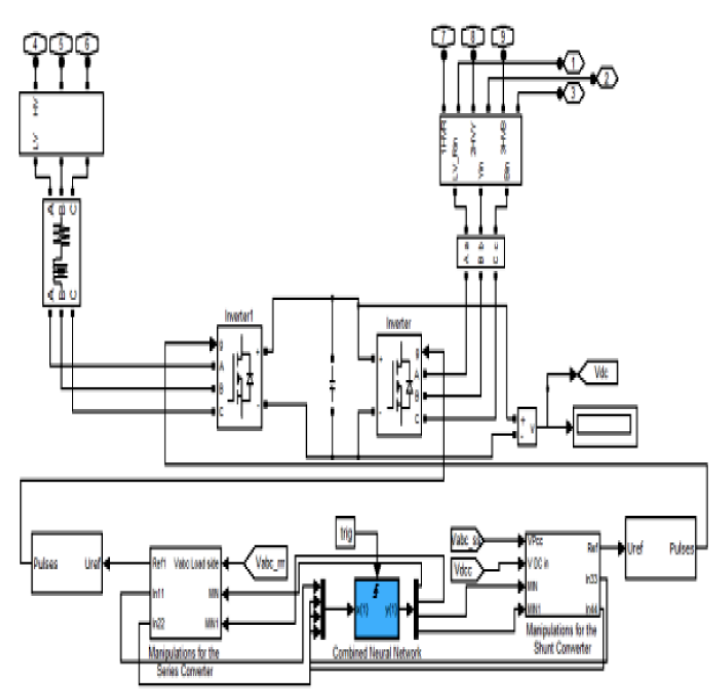

Figure 30. SIMULINK model with the combined single ANN controller.

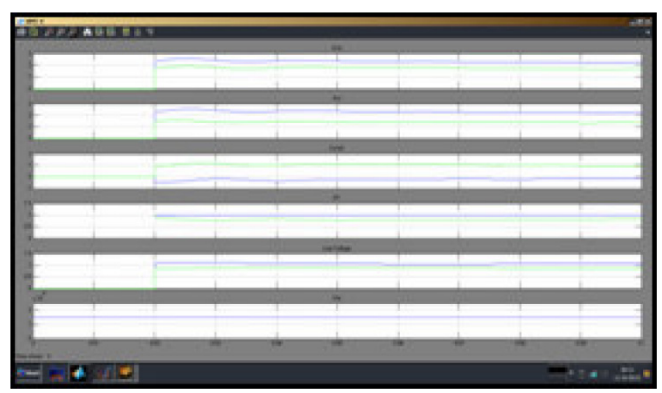

Figure 31. The comparison of the source side and load side parameters like KVA, KW,KVAR, PF and the DC link voltage.

and the actual voltage at PCC were all compared in the different control methodologies.

The comparison of the performance parameters has been given wide Table 1 .

The results, as depicted in the graphs and the table given, reveal that the novel single Neural Network based controller outperforms all other techniques compared in this paper and as such it is suggested.

That the combined ANN model be implemented in the real time applications. The combined ANN model of control can be implemented in real time using a 32 bit DSP or the ARM processor.

Trained ANN can be implemented in the $C$ language and can be down loaded in the micro controller in the form of HEX code.
Table 1. Comparison of the performance

\begin{tabular}{lcccc} 
parameters & PI & Fuzzy & $\begin{array}{c}\text { Four ANN } \\
\text { Unit }\end{array}$ & $\begin{array}{c}\text { Combined } \\
\text { ANN }\end{array}$ \\
\hline P to Grid & 0.59 & 0.6 & 0.6 & 0.6 \\
Q to Grid & 0.3 & 0.3 & 0.3 & 0.3 \\
PF at Source & 0.96 & 0.97 & 0.97 & 0.97 \\
& & & & \\
THD at PCC & 1.2 & 0.8 & 0.4 & 0.12 \\
\hline
\end{tabular}

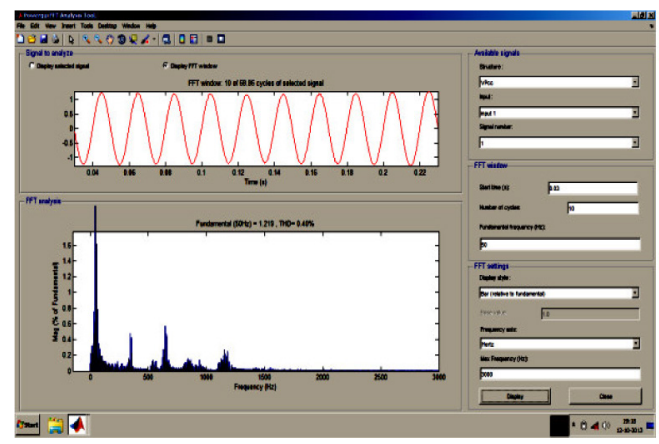

Figure 32. Spectrum of the voltage at PCC with combined ANN unit.

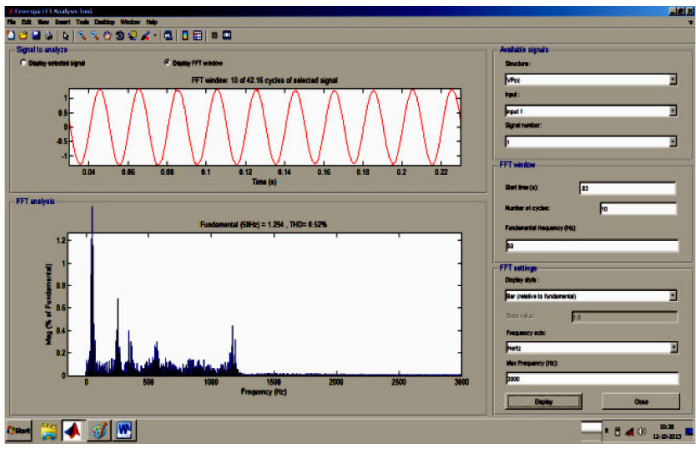

Figure 33. Spectrum of the voltage at the point of common coupling with four ANN units.

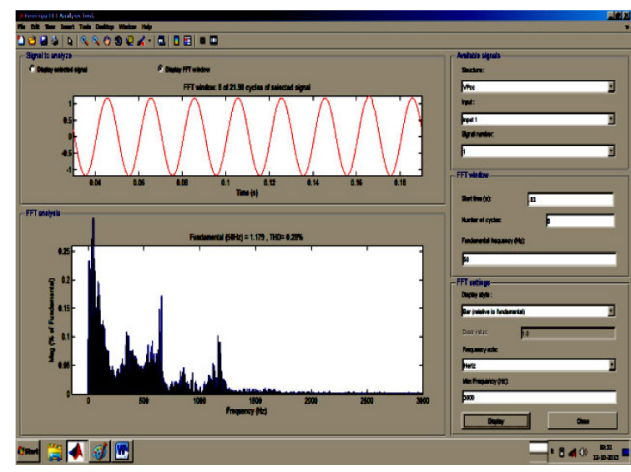

Figure 34. Spectrum of the Voltage at the point of common coupling using four FLC units. 


\section{Conclusion}

A single ANN based controller has been developed for the management of the UPFC. The results of simulation in the MATLAB / SIMULINK environment show that the method adopted is effective and can be implemented in real time applications.

\section{References}

1. Hingorani NG. High power electronics and flexible AC transmission system. IEEE Power Engineering Review. 1988 Jul; 3-4.

2. Gyugyi L. Unified power flow controller concept for flexible AC Transmission system. IEE Proceedings-C. 1992 Jul, 139(4):323-31.

3. Gyugyi L, Schauder CD, Williams SL, Rietman TR. The unified power flow controller: A new approach to power transmission control. IEEE Trans on Power Delivery. 1995 Apr; 10(2):1085-97.

4. Mishra S, Dash PK, Panda G. TS-fuzzy controller for UPFC in a multimachine power system. Proc. Inst Elect Eng Gen Transm Distrib. 2000; 147(1):15-22.

5. Yu Q, Norum L, Undeland T, Round S. Investigation of dynamic controllers for a unified power flow controller. Proc IEEE 22nd Int Conf Ind Electron Control Instrum, Taiwan; 1996 Aug; 1764-69.

6. Sreenvasachar K, Jayaram S, Salama MMA. Intelligent autonomous control of a unified power flowcontroller. Proc Int Conf Power Electron Drive Syst. 1997; 2:862-68.

7. Al-Awami A. A particle-swarm based approach of power system stability enhancement with UPFC. Elec Power Energy Syst. 2007; 29: 251-59.

8. Vilathgamuwa M, Choi San Shing,Tseng King Jet, Zhu Xin. A synchronous reference frame based control of a unified power flow controller. Proc Int Conf Power Electron Drive Syst. 1997; 2:844-49.
9. Farrag MEA, Putrus GA, Ran L. Advanced control of the unified power flow controller. Proc UPEC, Leicester, U.K., 1999, vol. 1, pp. 74-77. J. Shing and R. Jang, "ANFIS: Adaptive network-based fuzzy inference system," IEEE Trans. Syst., Man Cybern., vol. 23, no. 3, pp. 665-685, May/ Jun. 1993.

10. Edris A, Mehraban AS, Rahman M, Gyugyi L, Arabi S, Reitman T. Controlling the flow of real and reactive power. IEEE Comput.Appl. Power. 1998 Jan, 11(1):20-25.

11. Taher SA, Abrishami AA. UPFC location and performance analysis in deregulated power system. Math Problem Eng. 2009

12. Kumar A, Chanana S. DC model of UPFC and its use in competitive electricity market for loadability enhancement. Presented at the World Congr Eng Comput Sci. 2008 Oct 22-24; San Francisco.

13. Liu JY, Song YH. Determining maximum regulating capability of UPFC based on predicting feasibility limit of power systems. Elect Mach Power Syst. 1998; 20:789-800.

14. Bukley JJ. Theory of fuzzy controllers. Fuzzy Sets Syst. 1992; 51:249-58.

15. Alturki FA, Abdennour A. Design and simplification of adaptive neuro-fuzzy inference controllers for power plants. Elect Power Energy Syst. 1999; 21: 465-74.

16. Fujita $H$, Watanabe $Y$, Akagi $H$. Transient analysis of a unified power flow controller and its application to design of the dc-link capacitor. IEEE Trans Power Electron. 2001 Sep; 16(5):735-40.

17. DSPACE-ds1103 Controller Board. Features Reference; 2001 Jan.

18. Shahir FM, Babaei E. Dynamic modeling of UPFC by Two shunt voltage- source converters and a series capacitor. JCEE. 2013; 5(5):476-81.

19. Shing J, Jang R. ANFIS: Adaptive network-based fuzzy inference system. IEEE Trans Syst, Man Cybern. 1993 May/ Jun; 23(3):665-85. 